\title{
Incoherently generated coherences
}

\author{
Raisa I. Karasik*, Karl-Peter Marzlin ${ }^{\dagger, * *}$, Barry C. Sanders** and K. \\ Birgitta Whaley*, \\ *Applied Science \& Technology and Berkeley Quantum Information Center, University of \\ California, Berkeley, California 94720, USA \\ ${ }^{\dagger}$ Department of Physics, St Francis Xavier University, Antigonish, Nova Scotia B2G 2W5, Canada \\ ** Institute for Quantum Information Science, University of Calgary, Calgary, Alberta T2N 1N4, \\ Canada \\ ${ }^{\star}$ Department of Chemistry, University of California, Berkeley, California 94720, USA
}

\begin{abstract}
We have discovered that, in specific cases, decoherence due to the interaction with the environment can be avoided by just introducing a special driving field for an open quantum system. We show that this phenomenon constitutes a new class of decoherence-free subspaces which are useful for passive preservation of quantum information and discuss a physical example.
\end{abstract}

Keywords: open systems, decoherence free subspaces

PACS: $03.67 . \mathrm{Pp}, 42.50 . \mathrm{Ct}$

\section{INTRODUCTION}

Encoding into decoherence-free subspaces (DFS) [1,2] protects quantum states from the effects of decoherence by exploiting certain symmetries in the decoherence processes. This encoding can be useful for quantum memory and may reduce the need for quantum error correction and has been subject to experimental tests $[3,4,5,6,7]$.

In this paper, we discuss a new type of DFS that is created through "'incoherently generated coherences"' (IGC). Such subspaces exist for certain open systems, where the dynamics of a quantum system can connive together with the interactions between the system and its environment in a special way to reduce decoherence. We provide an example of a physical system that supports such states.

\section{DECOHERENCE-FREE SUBSPACES}

We study DFS in open quantum systems whose evolution is given by a Markovian semigroup master equation. In this approach, an open quantum system $S$ is coupled to the environment $R$. Tracing over the degrees of freedom of the environment leads to a description of the system's dynamics in terms of the reduced density matrix $\rho$ which describes the state of the system $S$. The evolution of $\rho$ is given by the Markovian master equation

$$
\dot{\rho}=-i\left[\hat{H}_{\mathrm{eff}}, \rho\right]+L_{D}[\rho],
$$

where $\hat{H}_{\text {eff }}$ is the effective-system Hamiltonian that also includes energy level shifts due to the interaction with the environment. $L_{D}[\rho]$ is often called dissipative or decoherence 
part of the Markovian master equation and is given by the following expression

$$
L_{\mathrm{D}}[\rho]=\frac{1}{2} \sum_{l=1}^{M} \lambda_{l}\left(\left[\hat{J}_{l}, \rho \hat{J}_{l}^{\dagger}\right]+\left[\hat{J_{l}} \rho, \hat{J}_{l}^{\dagger}\right]\right),
$$

where $\lambda_{l}>0$ and $\hat{J}_{l}$ are Lindblad operators which describe all possible decoherence processes in a quantum system $S$.

DFS have been proposed as a collection of states that undergo pure unitary evolution $[1,2]$. Early works on DFS [2] interpreted Markovian master equation by saying that the first term in the equation $\left(\left[\hat{H}_{\mathrm{eff}}, \rho\right]\right)$ generates unitary evolution, whereas $L_{D}[\rho]$ generates non-unitary decoherening dynamics. With this interpretation, it would be natural to define DFS as follows:

DFS definition 1: a DFS corresponds to a collection of states that satisfy $L_{D}[\rho(t)]=0$ for all times $t$. Then the evolution of $\rho$ will be generated by $-i\left[\hat{H}_{\text {eff }}, \rho\right]$ alone, which is unitary.

\section{INCOHERENTLY GENERATED COHERENCES}

In Ref. [8] we have shown that $L_{D}[\rho] \neq 0$ is not a necessary condition generating unitary dynamics. For systems described by Markovian master equations, pure states undergo unitary dynamics when purity $\operatorname{Tr}\left[\rho^{2}(t)\right]$ is preserved throughout the evolution. This suggests a different definition for decoherence-free subspaces in Markovian systems:

DFS definition 2: A DFS $\mathscr{H}_{\mathrm{DFS}} \subset \mathscr{H}_{\mathrm{S}}$ is a collection of pure states $\rho(t)$ generated by $\mathscr{H}_{\text {DFS }}$ that fulfill $\partial_{t} \operatorname{Tr}\left[\rho^{2}(t)\right]=0$ for all $t$.

DFS definitions 1 and 2 are different in a subtle way. To shed light on this we introduce a new class of DFS states:

Definition of IGC states: States with incoherent generation of coherences (IGC) are states $\rho(t)$ that satisfy DFS definition 2 but not DFS definition 1 , so that $\partial_{t} \operatorname{Tr}\left[\rho^{2}(t)\right]=0$ with $L_{D}[\rho(t)] \neq 0$.

Hence, IGC states evolve coherently, but for the - generally decohering - effect of the dissipative part of the master equation cannot be neglected during their evolution. As we have shown in Ref. [8] this is only possible if the dissipative part does generate a coherent, instead of decohering, effect on IGC states. This is not possible for all decoherence models but can occur under special circumstances.

\section{Example}

One example of a system that can support IGC states is given by a two-level atom with ground state $|1\rangle$ and excited state $|2\rangle$ that interacts with the quantized radiation field. In this example the environment corresponds to the radiation field which is initially prepared in a squeezed vacuum state. The time evolution of this system is given by the Markovian master equation [9]

$$
\dot{\rho}=-i\left[\hat{H}_{D}, \rho\right]+\frac{\gamma}{2}\left[2 \hat{J} \rho \hat{J}^{\dagger}-\hat{J}^{\dagger} \hat{J} \rho-\rho \hat{J}^{\dagger} \hat{J}\right],
$$


where the Lindblad operator take the form $\hat{J}=c \sigma_{-}+s \sigma_{+}$with $c \equiv \cosh (r), s \equiv \sinh (r)$ and $r$ is the real squeezing parameter. $\sigma_{+}$and $\sigma_{-}$are, respectively, the raising and lowering operators for the two-level atom, and $\gamma$ is the decay rate of the two-level atom in vacuum. The reduced density matrix of the two-level corresponds to a Hermitean $2 \times 2$ matrix with matrix elements $\rho_{i j}(t), i, j=1,2$.

If the system Hamiltonian takes the form

$$
\hat{H}_{D}=\frac{\gamma}{2} \sqrt{s c}(s-c) \hat{\sigma}_{y}=\frac{\gamma_{0}}{2} \sqrt{s c}(s-c)\left(\begin{array}{cc}
0 & -i \\
i & 0
\end{array}\right)
$$

then the general solution of Eq. (3) is given by

$$
\begin{aligned}
\rho_{11}(t)= & \frac{s}{s+c}+\frac{\sqrt{s c}(s-c)\left(\rho_{12}(0)+\rho_{21}(0)\right)}{s^{2}-6 s c+c^{2}}\left(\mathrm{e}^{-4(s-c)^{2} t}-\mathrm{e}^{-2(s+c)^{2} t}\right) \\
& -\frac{s}{s+c}\left(\frac{s^{2}-c^{2}}{s^{2}-6 s c+c^{2}} \mathrm{e}^{-4(s-c)^{2} t}+\frac{2 c(c-3 s)}{s^{2}-6 s c+c^{2}} \mathrm{e}^{-2(s+c)^{2} t}\right) \\
& +\left(\frac{(s-c)^{2}}{s^{2}-6 s c+c^{2}} \mathrm{e}^{-4(s-c)^{2} t}-\frac{4 s c}{s^{2}-6 s c+c^{2}} \mathrm{e}^{-2(s+c)^{2} t}\right) \rho_{11}(0) \\
\rho_{12}(t)= & \rho_{21}^{*}(t) \\
\rho_{21}(t)= & \frac{\sqrt{s c}}{s+c}-\frac{\sqrt{s c}}{s+c}\left(\frac{(s-c)(3 s-c)}{s^{2}-6 s c+c^{2}} \mathrm{e}^{-2(s+c)^{2} t}-\frac{2 s(s+c)}{s^{2}-6 s c+c^{2}} \mathrm{e}^{\left.-4(s-c)^{2} t\right)}\right) \\
& +\frac{2 s c}{s^{2}-6 s c+c^{2}}\left(\mathrm{e}^{-2(s+c)^{2} t}-\mathrm{e}^{-4(s-c)^{2} t}\right) \rho_{12}(0) \\
& +\left(\frac{s^{2}-4 s c+c^{2}}{s^{2}-6 s c+c^{2}} \mathrm{e}^{-2(s+b)^{2} t}-\frac{2 s c}{s^{2}-6 s c+c^{2}} \mathrm{e}^{-4(s-c)^{2} t}\right) \rho_{21}(0) \\
& +\frac{2 \sqrt{s c}(s-c)}{s^{2}-4 s c+c^{2}}\left(\mathrm{e}^{-2(s+c)^{2} t}-\mathrm{e}^{-4(s-c)^{2} t}\right) \rho_{11}(0) \\
\rho_{22}(t)= & -\rho_{11}(t) .
\end{aligned}
$$

This implies that the pure state $|\psi(t)\rangle=\sqrt{s}|2\rangle+\sqrt{c}|1\rangle$, with corresponding density matrix

$$
\rho(t)=|\psi\rangle\langle\psi|=\frac{1}{s+c}\left(\begin{array}{cc}
s & \sqrt{c s} \\
\sqrt{c s} & c
\end{array}\right)
$$

solves Eqs. (5-8) and thus indeed undergoes a (trivial) unitary evolution. Furthermore,

$$
L_{D}[\rho(t)]=\frac{\gamma}{2} \frac{s-c}{s+c}\left(\begin{array}{cc}
2 c s & \sqrt{c s}(s-c) \\
\sqrt{c s}(s-c) & 2 c s
\end{array}\right) \neq 0
$$

so that $|\psi(t)\rangle$ corresponds to an IGC state: it undergoes unitary evolution even though the decoherence term of the Markovian master equation is not zero.

The interplay between the dissipative part and the coherent part of the master equation is critical for the existence of IGC states. To demonstrate this, we consider the case when the driving Hamiltonian is absent from the master equation, $\hat{H}_{D}=0$. The general solution 
to Eq. (3) is then given by

$$
\begin{aligned}
& \rho_{11}(t)=\frac{s^{2}}{s^{2}+c^{2}}+\left(\rho_{11}(0)-\frac{s^{2}}{s^{2}+c^{2}}\right) \mathrm{e}^{-4\left(s^{2}+c^{2}\right) t} \\
& \rho_{12}(t)=\frac{\rho_{12}(0)+\rho_{21}(0)}{2} \mathrm{e}^{-2(s-c)^{2} t}+\frac{\rho_{12}(0)-\rho_{21}(0)}{2} \mathrm{e}^{-2(s+c)^{2} t} \\
& \rho_{21}(t)=\frac{\rho_{12}(0)+\rho_{21}(0)}{2} \mathrm{e}^{-2(s-c)^{2} t}-\frac{\rho_{12}(0)-\rho_{21}(0)}{2} \mathrm{e}^{-2(s+c)^{2} t} \\
& \rho_{22}(t)=\frac{c^{2}}{s^{2}+c^{2}}+\left(\rho_{22}(0)-\frac{c^{2}}{s^{2}+c^{2}}\right) \mathrm{e}^{-4\left(s^{2}+c^{2}\right) t}
\end{aligned}
$$

so that all states decay to

$$
\rho(t \rightarrow \infty)=\left(\begin{array}{cc}
\frac{s^{2}}{s^{2}+c^{2}} & 0 \\
0 & \frac{c^{2}}{s^{2}+c^{2}}
\end{array}\right) .
$$

Thus, without driving all pure states decay into incoherent mixture of ground and excited state. We see that in this system the application of a specific driving Hamiltonian can eliminate decoherence for IGC states.

In conclusion, we have introduced a new class of decoherence-free states for which the effect of the dissipative part of the master equation is crucial to achieve coherence. In the example presented above in Eqs. (5-8), one has $L_{D}[\rho(t)]=i\left[\hat{H}_{D}, \rho(t)\right]$, so that the driving field negates the decohering effects of $L_{D}[\rho]$ and allows to preserve the state.

\section{ACKNOWLEDGMENTS}

This project has been financially supported by iCORE, NSERC, CIFAR. R.I.K. and K.B.W. thank the NSF for financial support under ITR Grant No. EIA-0205641, and the Defense Advanced Research Projects Agency (DARPA) and the Air Force Laboratory, Air Force Material Command, USAF, under Contract No. F30602-01-2-0524.

\section{REFERENCES}

1. P. Zanardi and M. Rasetti, Phys. Rev. Lett. 79, 3306 (1997).

2. D. A. Lidar, I. L. Chuang and K. B. Whaley, Phys. Rev. Lett. 81, 2594 (1998).

3. P. G. Kwiat, A. J. Berglund, J. B. Altepeter, and A. G. White, Science 290, 498 (2000).

4. D. Kielpinski, et al., Science 291, 1013 (2001).

5. L. Viola, et al., Science 293, 2059 (2001).

6. M. Mohseni, J. S. Lundeen, K. J. Resch, and A. M. Steinberg, Phys. Rev. Lett. 91, 187903 (2003).

7. C. Langer, et al., Phys. Rev. Lett. 95, 060502 (2005).

8. R. Karasik, K.-P. Marzlin, B. C. Sanders and K. B. Whaley, Phys. Rev. A 77(5), 052301 (2008).

9. H.-P. Breuer and F. Petruccione, The Theory of Open Quantum Systems (Oxford University Press, Oxford, 2002). 